\title{
ON SOME ROBUSTNESS ISSUES IN INPUT DESIGN
}

\author{
Håkan Hjalmarsson and Jonas Mårtensson and Bo Wahlberg
}

\author{
Department of Signals, Sensors and Systems, KTH \\ SE-100 44 Stockholm, Sweden \\ hakan.hjalmarssones3.kth.se
}

\begin{abstract}
It is commonly believed that solutions to optimal input design problems for identification of dynamical systems often are sensitive to the underlying assumptions. For example, a wide class of problems can be solved with sinusoidal inputs with the same number of excitation frequencies (over the frequency range $(-\pi, \pi]$ ) as number of estimated parameters. With such an input it is not possible to check whether the true system is of higher order or not since then the input is not persistently exciting enough. In this contribution we argue that the optimal solution is often not unique and that there are alternatives to sinusoidal inputs which are more robust. For simplicity, we restrict attention to finite impulse-response models. For such a model of order $n$, it is only the $n$ first auto-correlation coefficients of the input which determine the accuracy of the parameter estimate. Thus, the remaining coefficients can be used to make the solution more robust. For the problem of estimating some scalar system quantity $J$ with a prescribed accuracy using minimum input energy, there is, under certain assumptions, an input spectrum that is optimal regardless of the model order. Furthermore, we show that using this input allows $J$ to be estimated consistently even when the model order is lower than the true system order. Copyright (c) 2006 IFAC
\end{abstract}

Keywords: Input design, model error, under-modeling.

\section{INTRODUCTION}

Judicious choice of the input excitation is crucial for successful identification of dynamical systems. There is an extensive literature on optimal input design. A standard approach has been to focus on model quality measures which are functions of the asymptotic covariance matrix of the parameter estimates. In the 1970s significant contributions to this area were made, see e.g. (Fedorov, 1972), (Mehra, 1974), (Goodwin and Payne, 1977), and (Zarrop, 1979), and it was realized that many optimal input design problems can be solved using sinusoidal inputs. This originated from the result that all achievable information matrices $P^{-1}\left(\theta_{o}\right)$ can be constructed using inputs comprising a finite number of sinusoids (Mehra, 1974). Typically it is sufficient to use the same number of excitation frequencies (over the interval $(-\pi, \pi])$ as the number of parameters to be estimated. While such types of

\footnotetext{
1 This work was supported by the Swedish Research Council
}

solutions are elegant and provide significant insight into the optimal input design problem, their utility in practice is limited. The optimal input typically depends on unknown system properties which have to be pre-determined in the design. In particular the true system order is typically unknown. Hence, an input comprising of a sum of sinusoids may turn out to be persistently exciting of too low order when the collected data is analyzed. This limitation seems to indicate that optimal inputs are sensitive to the underlying assumptions. One approach to avoid this has been to make designs based on the properties of high order models (Ljung, 1985; Ljung and Yuan, 1985; Yuan and Ljung, 1985; Gevers and Ljung, 1986; Hjalmarsson et al., 1996; Ljung, 1999; Zhu and van den Bosch, 2000; Forssell and Ljung, 2000).

The main objective of this paper is to highlight that often there are additional degrees of freedom in the properties of the input that can be used to ensure different robustness aspects of the design. 


\section{PROBLEM SET-UP AND A PREVIEW}

For simplicity of the presentation we will restrict our attention to finite impulse-response (FIR) systems

$$
\begin{aligned}
y(t) & =\sum_{k=0}^{n_{o}} b_{k}^{o} u(t-k)+e_{o}(t)=\left[\theta_{n_{o}}^{o}\right]^{T} \Gamma_{n_{o}}(q) u(t)+e_{o}(t) \\
& =G\left(q, \theta_{n_{o}}^{o}\right) u(t)+e_{o}(t)
\end{aligned}
$$

where $\Gamma_{n}(q)=\left[1, q^{-1}, \cdots, q^{-n}\right]^{T}$ with $q^{-1}$ denoting the backward time shift operator, where $\theta_{n_{o}}^{o}=$ $\left[b_{o}^{o}, \ldots, b_{n_{o}}^{o}\right]^{T}$ and where $e_{o}(t)$ is zero mean white noise with variance $\sigma_{o}^{2}$. Correspondingly, model structures of the type

$$
y(t)=\sum_{k=0}^{n} b_{k} u(t-k)+e(t)=\theta_{n}^{T} \Gamma_{n}(q) u(t)+e(t)(2)
$$

will be used.

Given input/output data $\{y(t), u(t)\}_{t=1}^{N}$ from the system, the model parameters are estimated using the prediction error criterion

$$
\begin{aligned}
& \hat{\theta}_{N, n}=\arg \min _{\theta_{n}} V\left(\theta_{n}\right), \\
& V\left(\theta_{n}\right)=\frac{1}{N} \sum_{t=1}^{N}\left[y(t)-\theta_{n}^{T} \Gamma_{n}(q) u(t)\right]^{2} .
\end{aligned}
$$

The components of the parameter estimate $\hat{\theta}_{N, n}$ are denoted by $\hat{b}_{n, N}^{k}$. When $n \geq n_{o}$, the associated asymptotic covariance matrix of the model error is given by

$$
\lim _{N \rightarrow \infty} N \mathbf{E}\left[\left(\hat{\theta}_{n}-\theta_{n}^{o}\right)\left(\hat{\theta}_{n}-\theta_{n}^{o}\right)^{T}\right]=P_{n},
$$

where $\theta_{n}^{o}=\left[b_{0}^{o}, \ldots, b_{n_{o}}^{o}, 0, \ldots, 0\right]^{T}$, and

$$
P_{n}^{-1}=\frac{1}{2 \pi \sigma_{o}^{2}} \int_{-\pi}^{\pi} \Gamma_{n}\left(e^{j \omega}\right) \Gamma_{n}^{*}\left(e^{j \omega}\right) \Phi_{u}(\omega) \mathrm{d} \omega .
$$

Here $\Phi_{u}(\omega)$ is the spectrum of the input signal $u(t)$, which can be written as

$$
\Phi_{u}(\omega)=\sum_{k=-\infty}^{\infty} \mu_{k} e^{j \omega k}>0
$$

where $\mu_{k}=\mathrm{E}[u(t) u(t-k)]$ are the auto-correlations.

We are interested in determining an input which ensures an accurate estimate of some specific scalar system property $J=J\left(\theta_{n_{o}}^{o}\right)$, such as for example:

I) one impulse response coefficient: $J_{I}\left(\theta_{n_{o}}^{o}\right)=b_{k}^{o}$,

II) $\mathscr{H}_{2}$-gain: $J_{I I}\left(\theta_{n_{o}}^{o}\right)=\left\|G\left(q, \theta_{n_{o}}^{o}\right)\right\|_{2}^{2}=\sum_{k=0}^{n_{o}}\left|b_{k}^{o}\right|^{2}$,

III) the location of a particular zero $z_{o}$ (or pole) of the system: $J_{I I I}\left(\theta_{n_{o}}^{o}\right)=z_{o}$, or

IV) the static gain $J_{I V}\left(\theta_{n_{o}}^{o}\right)=\sum_{k=0}^{n_{o}} b_{k}^{o}$.

The corresponding estimate using (2) and (3) is given by $J\left(\hat{\theta}_{N, n}\right)$. Assuming that the system is in the model class, i.e. $n \geq n_{o}$, a reasonable measure of the model quality is then the asymptotic variance

$$
Q\left(\theta_{n_{o}}^{o}, n, \Phi_{u}\right)=\lim _{N \rightarrow \infty} N \mathbf{E}\left[\left|J\left(\hat{\theta}_{N, n}\right)-J\left(\theta_{n_{o}}^{o}\right)\right|^{2}\right]
$$

which, as will be shown below, depends on the input through its spectrum $\Phi_{u}$ only.
Problem 1. The specific problem we will consider is that of finding an input which has the least power such that $Q\left(\theta_{n_{o}}^{o}, n, \Phi_{u}\right) \leq \gamma$, i.e.

$$
\begin{aligned}
& \underset{\Phi_{u}}{\operatorname{minimize}} \frac{1}{2 \pi} \int_{-\pi}^{\pi} \Phi_{u}(\omega) \mathrm{d} \omega \\
& \text { subject to } Q\left(\theta_{n_{o}}^{o}, n, \Phi_{u}\right) \leq \gamma,
\end{aligned}
$$

when the true system is in the model class, i.e. when $n \geq n_{o}$.

Example 2.1. (Impulse response coefficient). Consider the problem of estimating the first impulse response coefficient $b_{0}^{o}$, cf. I) above.

$\left[\mathbf{n}=\mathbf{n}_{\mathbf{0}}\right]$ Assume first that $n=n_{o}$. Our performance measure $Q\left(\theta_{n_{o}}^{o}, n_{o}\right)$ is in this case the $(1,1)$-element of the asymptotic covariance matrix $P_{n}$. For a static system, i.e. when $n_{o}=0, P_{n}$ is scalar and equal to $\sigma_{o}^{2} / \mu_{0}$. Hence, it is obvious that any input having $\mu_{0}=$ $\frac{\sigma_{o}^{2}}{\gamma}$ is optimal. Thus in this case there is considerable freedom in the choice of input spectrum. For example, a constant input with amplitude $\sqrt{\sigma_{o}^{2} / \gamma}$ is optimal.

However, the choice of a white input is a particularly interesting choice since then for multi-parameter problems $P_{n}=\frac{\sigma_{o}^{2}}{\mu_{0}} I_{n}$, i.e. the parameter estimates become uncorrelated. Hence, a white input with variance $\sigma_{o}^{2} / \mu$ guarantees the constraint in (7) regardless of the true system order $n_{o}$ ! In fact a white input is optimal for any order $n_{0}$, when $n=n_{o}$, since the variance of individual parameter estimates is a non-decreasing function of the model order.

$\left[\mathbf{n}>\mathbf{n}_{\mathbf{o}}\right]$ Notice also that $P_{n}=\frac{\sigma_{o}^{2}}{\mu_{0}} I_{n}$ still holds when the model order is strictly larger than the system order so that the constraint in (7) is satisfied also in this case. Hence, this input allows for over-modeling without any performance degradation.

[n $<\mathbf{n}_{\mathbf{0}}$ ] The white input has yet another interesting property for this problem. Regardless of $n_{o}$, also models of restricted complexity, i.e. with $n<n_{o}$, will produce consistent estimates of the first impulse response coefficient. In particular a static model can be used. The variance of the estimate will now be higher due to the unmodelled dynamics but the point is that consistency still holds. The reason for this is again that a white input allows the impulse response coefficients to be estimated independently.

The example illustrates that a judicious choice of input signal spectrum can make the model accuracy, as measured by $Q\left(\theta_{n_{o}}^{o}, n, \Phi_{u}\right)$, insensitive to

i) the system order $n_{o}$,

ii) over-modeling $\left(n>n_{o}\right)$, and

iii) under-modeling $\left(n<n_{o}\right)$.

In this contribution we will elaborate on which properties $J$ this holds for and how the input should be chosen. We illustrate what can be achieved with two less trivial examples.

Example 2.2. ( $\mathscr{H}_{2}$-gain). Consider $J_{I I}$, i.e. the estimation of the squared $\mathscr{H}_{2}$-gain. Let $b_{k}^{o}, k=0,1, \ldots$ be a real-valued sequence. Suppose that 


$$
\Phi_{G_{\infty}^{o}}(\omega) \triangleq \sum_{k=-\infty}^{\infty} b_{|k|}^{o} e^{-j \omega k} \geq 0, \forall \omega
$$

Then:

$\left[\mathbf{n}=\mathbf{n}_{\mathbf{o}}\right]$ The input spectrum $\Phi_{u}(\omega)=\frac{2 b_{o}^{o} \sigma_{o}^{2}}{\gamma} \Phi_{G_{\infty}^{o}}(\omega)$ is an optimal solution to (7) for all systems of the form

$$
G\left(e^{j \omega}, \theta_{n_{o}}^{o}\right)=\sum_{k=0}^{n_{o}} b_{k}^{o} e^{-j \omega k}, n_{o}=0,1, \ldots
$$

when $n=n_{o}$.

[n $>\mathbf{n}_{\mathbf{0}}$ ] When $b_{k}^{o}=0$ for $k>n_{o}$, the quality measure $Q\left(\theta_{n_{o}}^{o}, n, \Phi_{u}\right)$ is independent of the model order $n$ for $n \geq n_{o}$ if the true system is given by (8) for some finite $n_{o}$.

[n $<\mathbf{n}_{\mathbf{0}}$ ] Any scalar multiple of the input spectrum in i) ensures that $\sum_{k=0}^{n} \hat{b}_{N, n}^{k} b_{k}^{o}$ is a consistent estimate of $\sum_{k=0}^{n_{o}}\left(b_{k}^{o}\right)^{2}$ when $0 \leq n<n_{o}$. In particular, notice that when $n=0$, only knowledge of the first true impulse reponse coefficient is required for a consistent estimate of the $\mathscr{H}_{2}$-gain.

Example 2.3. (Non-minimum phase zero). Consider $J_{I I I}$ with $z_{o}$ being a real-valued non-minimum phase zero of the system $G\left(q, \theta_{n_{o}}^{o}\right)$. Then:

$\left[\mathbf{n}=\mathbf{n}_{\mathbf{o}}\right]$ The input spectrum

$$
\Phi_{u}(\omega)=\frac{\sigma_{o}^{2}\left|z_{o}\right|^{2}}{\gamma\left|\widetilde{G}\left(z_{o}, \theta_{n_{o}}^{o}\right)\right|^{2}} \frac{1-\left|z_{o}\right|^{-2}}{\left|1-z_{o}^{-1} e^{-j \omega}\right|^{2}}
$$

where $\widetilde{G}\left(q, \theta_{n_{o}}^{o}\right)=\frac{G\left(q, \theta_{n_{o}}^{o}\right)}{1-z_{o} q^{-1}}$, is an optimal solution to (7). Notice that this spectrum is the same for all systems having a zero at $z_{o}$, save for a constant factor which depends on the gain at $z_{o}$ of the system when the zero at $z_{o}$ has been removed.

[n $\left.>\mathbf{n}_{\mathbf{0}}\right]$ The input spectrum (9) ensures that the quality measure $Q\left(\theta_{n_{o}}^{o}, n, \Phi_{u}\right)$ is independent of the model order $n$ for $n \geq n_{o}$.

[n $<\mathbf{n}_{\mathbf{0}}$ ] Any scalar multiple of the input spectrum (9) ensures that the zero $z_{o}$ is estimated consistently for all model structures possessing at least one zero. This result was first presented in (Mårtensson et al., 2005).

\section{MAIN RESULTS}

Using a Taylor expansion of $J(\theta)$ around the true parameter vector, the quality measure $Q\left(\theta_{n_{o}}^{o}, n, \Phi_{u}\right)$ can be expressed as

$$
Q\left(\theta_{n_{o}}^{o}, n, \Phi_{u}\right)=\Lambda_{n}^{*}\left(\theta_{n}^{o}\right) P_{n} \Lambda_{n}\left(\theta_{n}^{o}\right)
$$

where $\Lambda_{n}\left(\theta_{n}\right)=\frac{\mathrm{d} J\left(\theta_{n}\right)}{\mathrm{d} \theta_{n}}$ whose elements will be denoted by $\lambda_{k}\left(\theta_{n}\right), k=0, \ldots, n$. Thus $J$ influences $Q$ only through its partial derivatives $\lambda_{k}\left(\theta_{n}^{o}\right)$.

We now present the main technical results.

Condition 3.1. The sequence $\left\{\lambda_{k}\right\}_{k=-\infty}^{\infty}$ is such that

$$
\lambda(\omega) \triangleq \sum_{k=-\infty}^{\infty} \lambda_{k} e^{j \omega k}
$$

can be factored as

$$
\lambda(\omega)=\Phi_{\lambda}(\omega) \sum_{k=0}^{n} \alpha_{k} e^{j \omega k}
$$

for some constants $\alpha_{0}, \ldots, \alpha_{n}$ and $\Phi_{\lambda}(\omega)>0, \forall \omega$.

Theorem 3.1. Let $\left\{\lambda_{k}\right\}_{k=-\infty}^{\infty}$ satisfy Condition 3.1, with $n, n \geq n_{o}$, being the model order. Then

$$
\Phi_{u}(\omega)=\frac{\sigma_{o}^{2}}{\gamma} \frac{1}{2 \pi} \int_{-\pi}^{\pi} \lambda(v) \mathrm{d} \nu \Phi_{\lambda}(\omega)
$$

satisfies the constraint to the input design problem (7) for any property $J$ such that $\Lambda_{n}\left(\theta_{n}^{o}\right)=\left[\lambda_{0}, \ldots, \lambda_{n}\right]^{T}$. Furthermore, (13) solves (7) when all but one $\alpha_{k}$ is zero.

Proof: See Appendix A.

Remark 3.1. It can be shown that when $\left\{\lambda_{k}\right\}_{k=-\infty}^{\infty}$ does not satisfy Condition 3.1,

$$
\Phi_{u}(\omega)=\frac{\sigma_{o}^{2}}{\gamma}\left|\sum_{k=0}^{\infty} \lambda_{k} e^{j \omega k}\right|^{2}
$$

ensures the quality constraint in (7).

Theorem 3.2. Let $\left\{\lambda_{k}\right\}_{k=-\infty}^{\infty}$ satisfy Condition 3.1, with $n, 0 \leq n<n_{o}$, being the model order. Then $\Phi_{u}=\alpha \Phi_{\lambda}$ for arbitrary $\alpha>0$ ensures that

$$
\lim _{N \rightarrow \infty}\left(\hat{\theta}_{N, n}\right)^{T} \Lambda_{n}=J\left(\theta_{n_{o}}^{o}\right)
$$

when the function $J$ is such that

$$
J\left(\theta_{n_{o}}^{o}\right)=\left(\theta_{n_{o}}^{o}\right)^{T} \Lambda_{n_{o}}
$$

where $\Lambda_{n}=\left[\lambda_{0}, \ldots, \lambda_{n}\right]^{T}$.

Remark 3.2. The elements of $\Lambda_{n}$ are allowed to be dependent of $\theta_{n_{o}}^{o}$.

Proof: See Appendix A.

To clarify the implications and use of these theorems we will revisit the examples in Section 2.

Example 3.1. (Example 2.1 revisited).

$\left[\mathbf{n}=\mathbf{n}_{\mathbf{0}}\right]$ and $\left[\mathbf{n}>\mathbf{n}_{\mathbf{o}}\right]$ With $J\left(\theta_{n_{o}}^{o}\right)=b_{o}^{o}$, we have that $\lambda_{k}\left(\theta_{n}^{o}\right)=\delta_{k}$ (Dirac's delta function) when $n \geq n_{o}$. Taking $\lambda(\omega)=\Phi_{\lambda}(\omega)=1$ in Theorem 3.1 gives that $\Phi_{u}(\omega)=\sigma_{o}^{2} / \gamma$ is optimal for any $n_{o}$ and any $n \geq n_{o}$.

[n $<\mathbf{n}_{\mathbf{0}}$ ] Furthermore, Theorem 3.2 gives that a consistent estimate of $b_{o}^{o}$ is obtained for any model order $n<n_{o}$ when a white input is used.

Example 3.2. (Example 2.2 revisited).

$\left[\mathbf{n}=\mathbf{n}_{\mathbf{o}}\right]$ With $J\left(\theta_{n_{o}}^{o}\right)=\sum_{k=0}^{n_{o}}\left|b_{k}^{o}\right|^{2}$, we have that $\lambda_{k}\left(\theta_{n}^{o}\right)=2 b_{k}^{o}$ for $k=0, \ldots, n_{o}$ and $\lambda_{k}\left(\theta_{n}^{o}\right)=0$ otherwise. Taking $\lambda(\omega)=\Phi_{\lambda}(\omega)=\Phi_{G_{\infty}^{o}}\left(e^{j \omega}\right)$ (which is positive by the assumption in Example 2.2) in Theorem 3.1 gives that $\Phi_{u}(\omega)=2 b_{o}^{o} \sigma_{o}^{2} / \gamma \Phi_{G_{\infty}^{o}}\left(e^{j \omega}\right)$ is optimal for any $n_{o}=n$.

[n $>\mathbf{n}_{\mathbf{0}}$ ] Consider now that $n_{o}$ is fixed and define $b_{k}^{o}=0, k=n_{o}+1, \ldots$ Then Theorem 3.1 gives the result in ii) in Example 2.2. 
[n $<\mathbf{n}_{\mathbf{0}}$ ] Furthermore, Theorem 3.2 gives that iii) in Example 2.2 holds.

Example 3.3. (Example 2.3 revisited).

$\left[\mathbf{n}=\mathbf{n}_{\mathbf{0}}\right]$ and $\left[\mathbf{n}>\mathbf{n}_{\mathbf{0}}\right]$ From (Lindqvist, 2001) we get that $\lambda_{k}\left(\theta_{n}^{o}\right)=\frac{z_{o}}{G\left(z_{o}, \theta_{n_{o}}^{o}\right)} z_{o}^{-k}$ for $n \geq n_{o}$. Since $\sum_{k=-\infty}^{\infty} z_{o}^{-|k|} e^{j \omega k}$ is positive when $\left|z_{o}\right|>1$, Theorem 3.1 gives that the results in i) and ii) in Example 2.3 holds.

$\left[\mathbf{n}<\mathbf{n}_{\mathbf{o}}\right]$ Now, consider instead $J\left(\theta_{n_{o}}^{o}\right)=\left(\theta_{n_{o}}^{o}\right)^{T} \Lambda_{n_{o}}\left(\theta_{n_{o}}^{o}\right)$ for which it holds that $J\left(\theta_{n_{o}}^{o}\right)=0$, since $\lambda_{k}\left(\theta_{n_{o}}^{o}\right) \equiv z_{o}^{-k}$ and $z_{o}$ is a zero of the system. Theorem 3.2 gives that the using a scalar multiple of the spectrum in i) in Example 2.3 will result in that $\left[1, z_{o}^{-1}, \ldots, z_{o}^{-n}\right] \theta_{n}^{*}=$ $J\left(\theta_{n_{o}}^{o}\right)=0$, i.e. $z_{o}$ becomes a zero of the estimated model as $N \rightarrow \infty$ when $n>0$.

Remark 3.3. Notice that $\left[\mathbf{n}<\mathbf{n}_{\mathbf{0}}\right]$ in Example 3.3 illustrates that Theorem 3.2 is applicable to properties that only implicitly are defined by $J$.

\section{ROBUSTNESS WITH RESPECT TO UNKNOWN SYSTEM PARAMETERS}

Unless the derivatives of $J$ are system independent, as for $J_{I}$, the optimal solution provided by Theorem 3.1 depends on the true unknown system. When it is known (or likely) that the true parameter vector $\theta_{n_{o}}^{o}$ lies in a specified set $\Theta$ and $\sum_{k=-\infty}^{\infty} \lambda_{k}\left(\theta_{n_{o}}^{o}\right) e^{j \omega k} \geq 0, \forall \omega, \forall \theta_{n_{o}}^{o} \in \Theta$, the spectrum

$$
\Phi_{u}(\omega)=\sup _{\theta_{n_{o}}^{o} \in \Theta} \frac{\sigma_{o}^{2}}{\gamma N} \lambda_{0}\left(\theta_{n_{o}}^{o}\right) \sum_{k=-\infty}^{\infty} \lambda_{k}\left(\theta_{n_{o}}^{o}\right) e^{j \omega k}
$$

will ensure that the quality constraint in (7) is satisfied. This input is therefore in some sense robust with respect to the true parameter value.

\section{ILLUSTRATION OF INPUT DESIGN}

In this section we will use a second order example to graphically illustrate how the estimates depend on the input spectrum and how the optimal solution looks like. Assume that the true system is a second order FIR-system given by

$$
y(t)=2 u(t)+u(t-1)+e_{o}(t)=\theta_{n}^{o} \Gamma_{n}(q) u(t)+e_{o}(t)
$$

where $e_{o}(t)$ is white noise with zero mean and variance 1. First consider a (full) second order FIR-model

$$
y(t)=b_{0} u(t)+b_{1} u(t-1)=\theta_{n}^{T} \Gamma_{n}(q) u(t)
$$

where $\theta_{n}=\left[b_{0}, b_{1}\right]^{T}$. In the mean, the parameter estimate $\hat{\theta}_{n}$ will be equal to the true parameter vector $\theta_{n}^{o}$ and the covariance matrix will be approximately $\frac{1}{N} P_{n}$. Now $N\left(\hat{\theta}_{n}-\theta_{n}^{o}\right)^{T} P_{n}^{-1}\left(\hat{\theta}_{n}-\theta_{n}^{o}\right)$ will be $\chi^{2}$-distributed and we can construct confidence regions for the parameter estimates on the form

$$
\left(\hat{\theta}_{n}-\theta_{n}^{o}\right)^{T} R_{n}\left(\hat{\theta}_{n}-\theta_{n}^{o}\right) \leq \alpha .
$$

These are ellipsoidal regions whose shape and size can be affected by changing $R_{n}$, i.e., by changing the input spectrum.
Suppose now that we want to estimate the static gain of the system (17) from measurements $\{y(t), u(t)\}_{t=1}^{250}$. First consider a white noise input. If we let the input power be normalized to 1 we get $R_{n}=\left[\begin{array}{ll}1 & 0 \\ 0 & 1\end{array}\right]$ which gives circular confidence regions. A $95 \%$ confidence region is shown in Figure 1a together with 100 parameter estimates based on simulations of the true system (17) with different realizations of the noise $e(t)$ and it is clear that about $95 \%$ of the parameter estimates does indeed lie inside the confidence region.

Now, the quantity of interest is not the parameters themselves but the static gain of the system. The static gain is equal to the sum of the parameters, i.e. $J\left(\theta_{n}\right)=b_{0}+b_{1}$. The static gain of the true system is $J\left(\theta_{n}^{o}\right)=3$ and the line $b_{0}+b_{1}=3$ is also shown in Figure 1a. All estimates on this line is equally good in our perspective.

So, let us see what happens when the optimal input signal is applied instead. The optimal input gives $R_{n}=\left[\begin{array}{ll}1 & 1 \\ 1 & 1\end{array}\right]$ which corresponds to confidence regions that are degenerate ellipses, infinitely stretched in one direction. To make the figures clearer, we use a nearoptimal input signal instead, given by $R_{n}=\left[\begin{array}{cc}1 & 0.98 \\ 0.98 & 1\end{array}\right]$. As shown in Figure 1c, the ellipses are shaped so that the uncertainty is large in the direction where $J\left(\theta_{n}\right)$ is constant. Figure 1c shows the same things as Figure 1a but for the optimal input signal.

This is easily extended to higher order systems. All systems in the hyperplane $\sum_{k=0}^{n} b_{k}=G\left(1, \theta_{n}^{o}\right)$ has the same static gain as the true system. The optimal input will be given by a matrix $R_{n}$ where all elements are equal to 1 . The confidence regions will be degenerate ellipsoids that are infinitely stretched in the directions of the hyperplane and has one finite axis perpendicular to the hyperplane.

\subsection{First order model}

We will now visualize what happens when we use a model with low complexity $\left(n<n_{o}\right)$, a first order model in this example. The model has only one parameter $y(t)=b_{0} u(t)$, but the aim is still to estimate the static gain of the true system, $J\left(\theta_{n_{o}}^{o}\right)=b_{0}^{o}+b_{1}^{o}$. The estimate of the static gain will be $J\left(\hat{\theta}_{n}\right)=\hat{b}_{0}$ but unlike the full order modeling case, the expected value of the estimate $J\left(\hat{\theta}_{n}\right)$ will depend on $R_{n}$ and it will typically deviate from $J\left(\theta_{n_{o}}^{o}\right)$.

Consider a full order model. The parameter estimate is given by $\hat{\theta}_{n}=\arg \min _{\theta_{n}} V\left(\theta_{n}\right)$, (cf. (3)). Letting $N \rightarrow \infty$ and taking expectation gives

$$
\begin{aligned}
\bar{V}\left(\theta_{n}\right) & =\overline{\mathbf{E}}\left\{\left(y(t)-G\left(q, \theta_{n}\right) u(t)\right)^{2}\right\} \\
& =\overline{\mathbf{E}}\left\{\left(\left(\theta_{n}^{o}-\theta_{n}\right)^{T} \Gamma_{n}(q) u(t)+e_{o}(t)\right)^{2}\right\} .
\end{aligned}
$$

Let $u(t)$ and $e_{o}(t)$ be uncorrelated and use Parseval's theorem to write

$$
\begin{aligned}
\bar{V}\left(\theta_{n}\right) & =\overline{\mathbf{E}}\left\{\left(\left(\theta_{n}^{o}-\theta_{n}\right)^{T} \Gamma_{n}(q) u(t)\right)^{2}\right\}+\sigma_{o}^{2} \\
& =\left(\theta_{n}^{o}-\theta_{n}\right)^{T} R_{n}\left(\theta_{n}^{o}-\theta_{n}\right)+\sigma_{o}^{2} .
\end{aligned}
$$

Compare with (19) and note that the ellipsoids are also level curves for $\bar{V}(\theta)$. For a full order model, the 


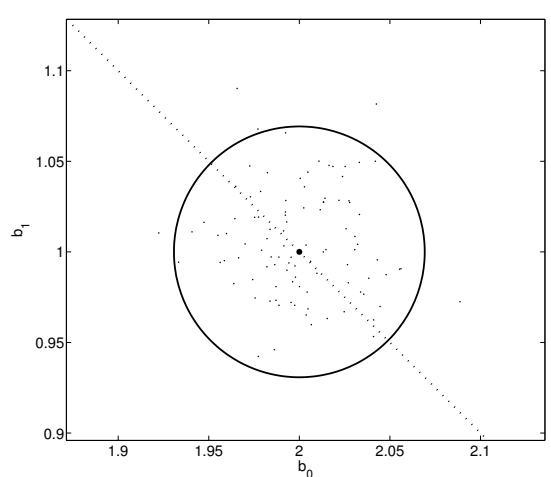

(a)

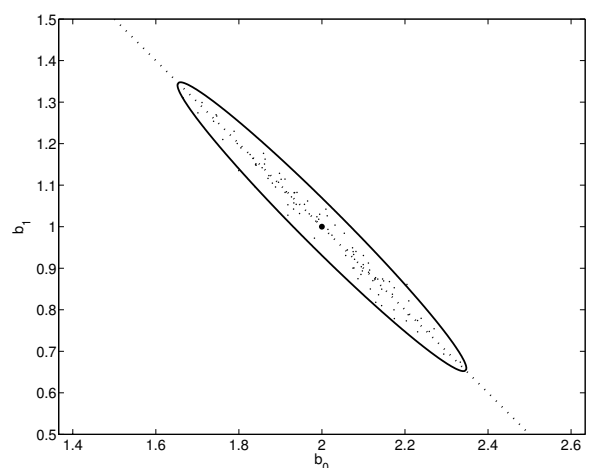

(c)

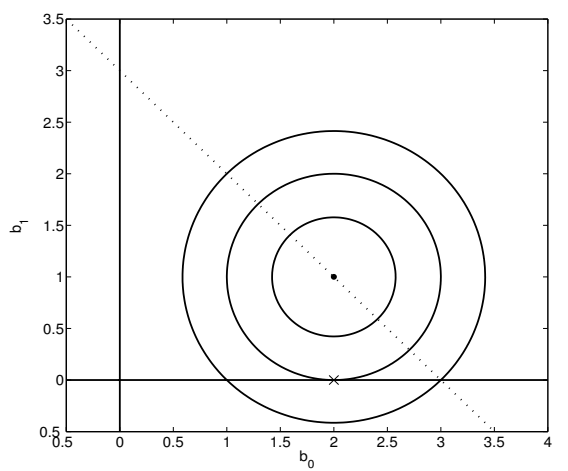

(b)

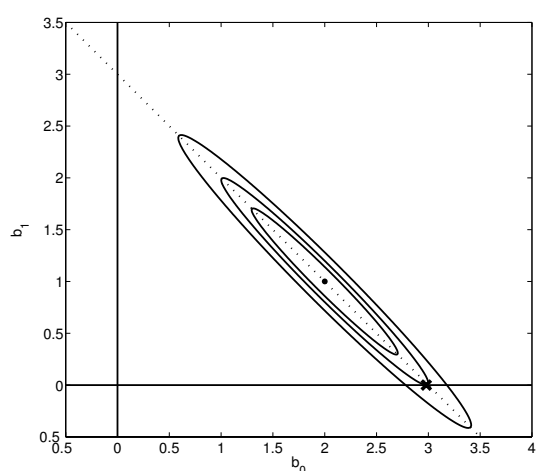

(d)

Fig. 1. In (a)-(d): The dotted line shows parameters where $J\left(\theta_{n}\right)=J\left(\theta_{n}^{0}\right)$. White noise input is used in (a)-(b) and (near)optimal input is used in (c)-(d). In (a) and (c): The circle/ellipse is the $95 \%$ confidence bound for the parameter estimate. The small dots are 100 parameter estimates where the system is simulated with different noise realizations. In (b) and (d): Circles/ellipses are level curves for $\bar{V}(\theta)$, see (20). The cross is the limiting parameter estimate for a first order model. More details are given in the text.

minimum is $\bar{V}\left(\theta_{n}^{o}\right)$ but for the low complexity model the parameter space is restricted to lie on the $\mathrm{x}$-axis since $b_{1}=0$.

In Figures $1 \mathrm{~b}$ and $1 \mathrm{~d}$ we see that the minimum along the $\mathrm{x}$-axis depends on the shape of the ellipses. When white input is used, the minimum is achieved for $b_{0}=$ 2 but when the optimal input signal is used, $\bar{V}(\theta)$ is minimized for $b_{o}=3$. Thus, the optimal design gives a correct estimate of the static gain also for a first order model.

\section{CONCLUSIONS}

In this contribution we have elaborated on some robustness issues in input design. It has been shown that there are degrees of freedom in the input spectrum that can be used to obtain an input signal that is optimal regardless of what the true system order is. Furthermore, we have shown that the accuracy becomes insensitive to over-modeling and finally, that this particular input allows consistent estimation of the system property of interest using models of lower order than the true system order. We find the latter result a very interesting observation and we believe that it can be generalized well beyond the somewhat restricted FIR scenario discussed above. If this is true, it will have far reaching implications since we in system identification always deal with model structures which cannot capture the entire system behavior. The result indicates that by choosing the input suitably we can estimate the desired quantity consistently regardless of the true sys- tem complexity. For a further discussion of this topic we refer to (Hjalmarsson, 2004).

\section{Appendix A. PROOFS OF THEOREMS 3.1-3.2}

Proof of Theorem 3.1: Due to lack of space, we will only prove the theorem when $\lambda(\omega)=\Phi_{\lambda}(\omega)$.

The quality constraint $Q \leq \gamma \Leftrightarrow \frac{\gamma}{\sigma_{o}^{2}}-\Lambda_{n}^{*} R_{n}^{-1} \Lambda_{n} \geq 0$ can be reformulated as a matrix inequality that is linear in $R_{n}$ by using Schur complements. View $\frac{\gamma}{\sigma_{o}^{2}}-$ $\Lambda_{n}^{*} R_{n}^{-1} \Lambda_{n} \geq 0$ as the Schur complement of $R_{n}$. The Schur complement being positive is equivalent to the matrix

$$
\left(\begin{array}{cc}
R_{n} & \Lambda_{n}\left(\theta_{n_{o}}^{o}\right) \\
\Lambda_{n}^{*}\left(\theta_{n_{o}}^{o}\right) & \frac{\gamma}{\sigma_{o}^{2}}
\end{array}\right) \geq 0
$$

being positive semi-definite. Now, taking the Schur complement of $\frac{\gamma}{\sigma_{o}^{2}}$, this is equivalent to

$$
R_{n}-\frac{\sigma_{o}^{2}}{\gamma} \Lambda_{n}\left(\theta_{n_{o}}^{o}\right) \Lambda_{n}^{*}\left(\theta_{n_{o}}^{o}\right) \geq 0
$$

which is linear in $R_{n}$. The input design problem can now be re-formulated as

$$
\begin{aligned}
& \underset{\mu_{0}, \ldots, \mu_{n}}{\operatorname{minimize}} \mu_{0} \\
& \text { subject to } R_{n}-\frac{\sigma_{o}^{2}}{\gamma} \Lambda_{n}\left(\theta_{n_{o}}^{o}\right) \Lambda_{n}^{*}\left(\theta_{n_{o}}^{o}\right) \geq 0 .
\end{aligned}
$$

The positivity of $\lambda$ implies that the Toeplitz matrix 


$$
T_{n}\left(\theta_{n_{o}}^{o}\right)=\left(\begin{array}{ccc}
\lambda_{0} & \cdots & \lambda_{n} \\
\vdots & \ddots & \vdots \\
\lambda_{n} & \cdots & \lambda_{0}
\end{array}\right) \geq 0
$$

is positive semi-definite. It follows that $\lambda_{0}^{2} \geq \lambda_{i}^{2}$ for $i=1 \ldots n$. Now a necessary condition for the constraint (A.2) to hold is that the diagonal elements are non-negative, i.e.

$$
\mu_{0} \geq \frac{\sigma_{o}^{2}}{\gamma} \max _{i} \lambda_{i}\left(\theta_{n_{o}}^{o}\right)^{2}=\frac{\sigma_{o}^{2}}{\gamma} \lambda_{0}^{2}\left(\theta_{n_{o}}^{o}\right)
$$

which means that we have a lower bound on the objective function $\mu_{0}$. The lower bound is obviously achieved with $\Phi_{u}(\omega)=\sigma_{o}^{2} / \gamma \lambda_{o} \lambda(\omega)$. That this spectrum satisfies the constraint (A.2) can be verified by first noting that the first row and column of (A.2) is zero. That the rest of the matrix is positive semidefinite can be shown by Schur complements again, together with the assumption that $T_{n} \geq 0$. This is a generalization of the results in (Mårtensson et al., 2005) for identification of non-minimum phase zeros and more details on that can be found in (Jansson, 2004).

Proof of Theorem 3.2: When $n<n_{o}, \hat{\theta}_{N, n}$ converges with probability one as $N \rightarrow \infty$ to the limit

$$
\theta_{n}^{*}=R_{n}^{-1} \frac{1}{2 \pi} \int_{-\pi}^{\pi} \Gamma_{n}\left(e^{j v}\right) \Phi_{u}(v) \Gamma_{n_{o}}^{*}\left(e^{j v}\right) \mathrm{d} v \theta_{n_{o}}^{o}
$$

where $R_{n}=\sigma_{o}^{2} P_{n}^{-1}$. We thus need to show that

$$
\begin{aligned}
& \left(\theta_{n_{o}}^{o}\right)^{T} \frac{1}{2 \pi} \int_{-\pi}^{\pi} \Gamma_{n_{o}}\left(e^{j v}\right) \Phi_{u}(v) \Gamma_{n}^{*}\left(e^{j v}\right) \mathrm{d} v R_{n}^{-1} \Lambda_{n}= \\
& \left(\theta_{n_{o}}^{o}\right)^{T} \Lambda_{n_{o}}
\end{aligned}
$$

or in other words that

$$
\frac{1}{2 \pi} \int_{-\pi}^{\pi} \Gamma_{n_{o}}\left(e^{j v}\right)\left|F_{u}\left(e^{j v}\right)\right|^{2} \Gamma_{n}^{*}\left(e^{j v}\right) \mathrm{d} v R_{n}^{-1} \Lambda_{n}=\Lambda_{n_{o}}
$$

where $F_{u}$ is a stable minimum phase factor of $\Phi_{u}$. Notice that

$$
\Lambda_{n}=\frac{1}{2 \pi} \int_{-\pi}^{\pi} \Gamma_{n}\left(e^{j \omega}\right) \lambda(\omega) \mathrm{d} \omega
$$

and hence the left-hand side of (A.6) can be expressed as

$$
\begin{aligned}
& \frac{1}{2 \pi} \int_{-\pi}^{\pi} \Gamma_{n_{o}}\left(e^{j v}\right)\left|F_{u}\left(e^{j v}\right)\right|^{2} \Gamma_{n}^{*}\left(e^{j v}\right) \mathrm{d} v R_{n}^{-1} \\
& \times \frac{1}{2 \pi} \int_{-\pi}^{\pi} \Gamma_{n}\left(e^{j \omega}\right) \lambda(\omega) \mathrm{d} \omega \\
& =\frac{1}{2 \pi} \int_{-\pi}^{\pi} \Gamma_{n_{o}}\left(e^{j v}\right) F_{u}\left(e^{j v}\right) \\
& \times \frac{1}{2 \pi} \int_{-\pi}^{\pi}[\underbrace{F_{u}^{*}\left(e^{j v}\right) \Gamma_{n}^{*}\left(e^{j v}\right) R_{n}^{-1} \Gamma_{n}\left(e^{j \omega}\right) F_{u}\left(e^{j \omega}\right)}_{\text {reproducing kernel } K(\omega, v)} \\
& \left.\times \frac{\lambda(\omega)}{F_{u}\left(e^{j \omega}\right)} \mathrm{d} \omega \mathrm{d} v\right] \\
& =\frac{1}{2 \pi} \int_{-\pi}^{\pi} \Gamma_{n_{o}}\left(e^{j v}\right) F_{u}\left(e^{j v}\right) \overline{\left\langle\frac{\lambda^{*}(\omega)}{F_{u}^{*}\left(e^{j \omega}\right)}, K(\omega, v)\right\rangle} \mathrm{d} v
\end{aligned}
$$

where $K(\omega, v)$ is the reproducing kernel of the space $X_{n}$ spanned by the elements in $F_{u}\left(e^{j \omega}\right) \Gamma_{n}\left(e^{j \omega}\right)$. This kernel has the property that

$$
\langle g(\omega), K(\omega, v)\rangle \triangleq \frac{1}{2 \pi} \int_{-\pi}^{\pi} \overline{K(\omega, v)} g(\omega) \mathrm{d} \omega=g(v)
$$

when $g \in X_{n}$. Under assumption (12) and with $\Phi_{u}=$ $\alpha \Phi_{\lambda}$ we have that $\lambda^{*} /\left|F_{u}\right|^{2}=\sum_{k=0}^{n} \alpha_{k} e^{-j \omega n}$ and hence $\lambda^{*} / F_{u}^{*} \in X_{n}$. Using this in (A.8) and subsequently (A.7) proves (A.6).

\section{REFERENCES}

Fedorov, V. V. (1972). Theory of Optimal Experiments, volume 12 of Probability and Mathematical Statistics. Academic Press.

Forssell, U. and L. Ljung (2000). Some results on optimal experiment design. Automatica 36(5), 749756.

Gevers, M. and L. Ljung (1986). Optimal experiment designs with respect to the intended model application. Automatica 22(5), 543-554.

Goodwin, G.C. and R.L. Payne (1977). Dynamic System Identification: Experiment Design and Data Analysis, Volume 136 of Mathematics in Science and Engineering. Academic Press.

Hjalmarsson, H. (2004). From experiments to control. Automatica.

Hjalmarsson, H., M. Gevers and F. De Bruyne (1996). For model-based control design, closed loop identification gives better performance. Automatica 32(12), 1659-1673.

Jansson, H. (2004). Experiment design with applications in identification for control. Doctoral thesis. KTH. Stockholm, Sweden.

Lindqvist, K. (2001). On Experiment Design in Identification of Smooth Linear Systems. Licentiate thesis. Royal Institute of Technology. Stockholm, Sweden.

Ljung, L. (1985). Asymptotic variance expressions for identified black-box transfer function models. IEEE Transactions on Automatic Control AC30, 834-844.

Ljung, L. (1999). System Identification - Theory for the User. 2nd ed.. PTR Prentice-Hall. Upper Saddle River, N.J.

Ljung, L. and Z.D. Yuan (1985). Asymptotic properties of black-box identification of transfer functions. IEEE Transactions on Automatic Control AC-30, 514-530.

Mårtensson, J., H. Jansson and H. Hjalmarsson (2005). Input design for identification of zeros. In: Proceedings of the 16th IFAC World Congress on Automatic Control.

Mehra, R. K. (1974). Optimal input signals for parameter estimation in dynamic systems-survey and new results. IEEE Transactions on Automatic Control AC-19, 753-768.

Yuan, Z.D. and L. Ljung (1985). Unprejudiced optimal open loop input design for identification of transfer functions. Automatica 21(6), 697-708.

Zarrop, M. (1979). Design for Dynamic System Identification. Lecture Notes in Control and Information Sciences. Sci. 21. Springer Verlag, Berlin.

Zhu, Y. C. and P. P. J. van den Bosch (2000). Optimal closed-loop identification test design for internal model control. Automatica 36(8), 1237-1241. 